\title{
GAIA Level 1 Neonatal Respiratory Tract Infection
}

National Cancer Institute

\section{Source}

National Cancer Institute. GAIA Level 1 Neonatal Respiratory Tract Infection. NCI

Thesaurus. Code C127991.

GAIA Level 1 Neonatal Respiratory Tract Infection is defined by two criteria: first, a new or progressive or persistent infiltrate or shadowing or fluid in the intrapleural cavity or interlobar fissure on chest X-ray OR a recognized virus identified using a validated assay from an upper respiratory sample OR a recognized pathogen identified, using a validated method, from a normally sterile site; second, three or more of the following requirements must be met: a) Temperature greater than or equal to 37.5 degrees $C$ or less than 35.5 degrees C; b) T achypnea or nasal flaring or chest indrawing or grunting; c) Desaturations or increased oxygen requirements or increased ventilator requirements or oxygen saturation less than 95\%; d) Apnea; e) Increased respiratory secretions or increased suctioning requirements; f) Cough, wheeze, or crepitations; g) Increased CRP or procalcitonin. 